\title{
Identité en négociation
}

Pratiques participatives, politique de développement et conflit identitaire en Pays Basque « français "

Identity under negotiation: participative practices, politics of development and identity conflict in "french" basque country

\section{Sébastien Ségas}

\section{(2) OpenEdition}

\section{Journals}

Édition électronique

URL : http://journals.openedition.org/plc/814

DOI : $10.4000 /$ plc. 814

ISSN : 2117-5209

Éditeur

L'Harmattan

Édition imprimée

Date de publication : 1 janvier 2010

Pagination : 13-36

ISBN : 978-2-296-1141-3

ISSN : 1279-8657

Référence électronique

Sébastien Ségas, "Identité en négociation », Pouvoirs dans la Caraïbe [En ligne], 16 | 2010, mis en ligne le 06 mai 2011, consulté le 01 mai 2019. URL : http://journals.openedition.org/plc/814 ; DOI :

$10.4000 /$ plc. 814 


\title{
IDENTITE EN NEGOCIATION : \\ PRATIQUES PARTICIPATIVES, POLITIQUE DE DEVELOPPEMENT ET CONFLIT \\ IDENTITAIRE EN PAYS BASQUE «FRANÇAIS »*
}

\author{
Sébastien SEGAS \\ Maître de conférences de Science Politique \\ Université de Rennes II
}

\section{Introduction}

Le discours modernisateur, tel qu'il se développe de nos jours en France et également dans quelques pays d'Europe (Gaudin, 1999), fait de la recherche de la proximité, via la mise en place de dispositifs locaux de démocratie participative, la condition de l'efficacité et de la légitimité de l'action publique «moderne ». Ce discours fournit un cadre d'interprétation et d'interaction (Goffman, 1991) qui articule, autour de la valeur transversale du «développement local », les thématiques du territoire et de l'identité locale à celles du projet, de la participation, de l'expertise et de la contractualisation.

Ce cadre a été mobilisé en Pays basque «français » pour lancer un programme de développement. Celui-ci a débuté en 1992, sur l'initiative du sous-préfet de Bayonne, par une démarche de réflexion prospective intitulée «Pays basque 2010», pendant laquelle des élus, des représentants de l'administration publique et du monde socioprofessionnel ont, en s'appuyant sur le travail d'un bureau d'étude, établi des scénarios de développement. Cette démarche a abouti, en juillet 1994, à la création d'un Conseil de Développement du Pays basque (CD), assemblée dédiée au dialogue avec la société civile où la mouvance autonomiste est largement représentée, puis en février 1995 à celle du Conseil des Élus du Pays basque (CE). Ces deux structures, qui vont être reconnues comme la base «d'un pays de préfiguration » dans le cadre de la loi Pasqua de 1995, ont collaboré à l'élaboration d'un Schéma d'Aménagement du Pays basque en 1996 et 1997. Cette démarche a débouché en décembre 2000 sur la signature d'une convention spécifique Pays basque annexée au contrat de plan État-Région, censée financer la mise en œuvre du schéma ${ }^{1}$.

\footnotetext{
* L'auteur tient à remercier C. Barbosa, J. Daniel, P. Le Galès et C. Sorbets pour leurs commentaires.

${ }^{1}$ L'enquête de terrain sur laquelle se base cette communication s'est déroulée en 2000-2001.
} 
Nous aimerions ici souligner comment ce cadre d'action publique (policy) a pu constituer, non seulement pour les pouvoirs publics mais également pour les groupes autonomistes, une ressource politique (politics) activée dans des stratégies de mobilisation et/ou de légitimation dans un contexte marqué par un conflit identitaire. De fait, le cadre participatif du développement a fait, en Pays basque, l'objet d'appropriations concurrentielles, chaque partie s'engageant dans une compétition pour l'imposition du sens légitime à donner à l'action conduite.

Ce sont d'abord les pouvoirs publics qui ont saisi ce cadre afin d'alimenter une opération de reconstruction symbolique (Padioleau, 1982) de la question identitaire. Cet «acte symbolique» (Geertz, 1964) visait à endiguer la contestation autonomiste et la revendication d'un statut politique pour le territoire basque en déplaçant le problème identitaire du registre nationaliste vers le registre du développement local. Par ce glissement d'un registre social à un autre, que l'on peut assimiler à une opération de «traduction » (Callon, 1986; Lascoumes, 1994) ou de «recadrage » (Goffman, 1991), l'Etat cherchait à transformer la manière dont les autonomistes interprétaient la situation et leur relation au système politique afin de créer les conditions d'une «institutionnalisation de la contestation » (Hirschman, 1995) dans le champ de l'action publique.

Pour autant, les autonomistes n'ont pas renoncé à la contestation : au contraire, ils se sont à leur tour appropriés le cadre, et les ressources et opportunités symboliques qu'il offre, afin de renforcer leur propre position revendicative tout en obtenant des concessions ou des avantages en terme d'action publique. Les autonomistes ont ainsi mené leur propre opération symbolique, investissant la légitimité du cadre (sa connotation «moderne » et « experte ») dans la reconfiguration de la revendication statutaire afin de gagner de nouveaux alliés à leur lutte.

\footnotetext{
${ }^{2}$ On peut précisément définir la traduction comme le passage d'un registre ou d'un cadre symbolique à un autre afin de produire des effets de mobilisation (M. Callon parlerait « d'enrôlement ») ou de démobilisation (parole critique).
} 


\section{La revendication statutaire en Pays basque «français»: la question départementale}

L'histoire moderne ${ }^{3}$ de la revendication départementale est indissociable de celle du nationalisme basque au nord des Pyrénées. Entrer dans le détail de cette histoire, avec son lot de divisions et de créations de micro-chapelles, dépasse de loin l'ambition de cet article. Nous nous contenterons ici de rappeler que cette lutte politique nationaliste revêt (ou a revêtu) deux formes: a) la lutte armée, menée par des groupes comme Iparretarak qui refusent tout compromis et dont l'idéologie interne justifie le recours à le violence ; b) le recours aux «formes » (Tilly, 1986 ; Trom, 2001) légitimes du répertoire et du vocabulaire de la mobilisation (action collective, élection) pour construire un rapport de force favorable dans une négociation avec l'État français.

Historiquement, cette deuxième forme de lutte s'est structurée autour de la revendication de la création d'un « département basque » formulée dès le début des années 1960 par le mouvement Enbata. La formulation de cette revendication révélait alors le souci stratégique de ce mouvement de construire une revendication suffisamment modérée afin de maximiser les soutiens (dans la population) et de minimiser les réticences (de l'État français) sans pour autant renoncer à l'objectif final d'une autonomie politique beaucoup plus grande, la création d'un département basque étant conçue comme une étape sur le chemin de l'autonomie ${ }^{4}$. En effet, il s'agissait non pas de réclamer à l'État et aux décideurs locaux un statut dérogatoire qui remettrait en cause l'architecture politico-institutionnelle de la France, mais simplement, au moins dans un premier temps, la constitution d'une collectivité locale de droit commun issu de la scission du département actuel des Pyrénées-Atlantiques 5 .

\footnotetext{
${ }^{3}$ La création d'un département basque avait déjà été réclamée au XVIII ${ }^{\mathrm{e}}$ et au XIX ${ }^{\mathrm{e}}$ siècle.

${ }^{4}$ Ainsi en 1963, les dirigeants d'Enbata, dans une motion politique solennelle proclament : « le mouvement Enbata [...] propose au peuple basque : - dans un premier stade, et dans le respect de la Constitution et des Lois qui régissent la République Française, la création d'un département basque comprenant les trois provinces de Soule, Basse-Navarre et Labourd, département qui jouira d'un statut de la langue basque; - dans un second stade, et dans l'Europe unie, la formation d'une région politiquement, administrativement et culturellement autonome, rassemblant les Sept Provinces Basques, et fédérée aux autres entités européennes ». ${ }^{5}$ Le Pays basque représente moins de la moitié de la superficie du département des PyrénéesAtlantiques.
} 
Cependant, la stratégie de mobilisation adoptée par Enbata a rencontré un certain nombre de difficultés. Tout d'abord, des résultats électoraux très décevants dans les années $1960^{6}$, ainsi que la dissolution d'Enbata par l'État français en 1974, en raison de liens présumés avec ETA, ont très vite amené le mouvement autonomiste à privilégier la scène sociale plutôt que la scène politique et à investir, dans les années 1970, la vie associative, l'animation culturelle, le mouvement syndical (en particulier avec le syndicat agricole ELB) et coopératif, de sorte que le milieu autonomiste a fini par constituer un réseau social très bien implanté auxquels des entreprises de mobilisation extérieures ont tenté de se greffer afin d'enrôler des relais sociaux et des soutiens actifs à même d'augmenter leurs propres capacités de mobilisation.

Ainsi, la Chambre de Commerce et d'Industrie (CCI) de Bayonne, relais institutionnel des milieux économiques locaux a tenté, au milieu des années 1970, de séduire le monde autonomiste pour élargir les soutiens à son propre projet de création de département. Très différente à son départ en 1974 de la revendication d'Enbata, le «nouveau département » réclamé par la CCI s'inscrivait non pas dans la perspective d'une «lutte pour la reconnaissance » d'une identité mais dans une « logique de conflit d'intérêts » (Honneth, 2000), le futur département étant censé assurer une meilleure prise en compte et une «médiation » (Crozier, Friedberg, 1977) des intérêts économiques du Pays basque par le politique : " pour les autonomistes, il s'agit là d'une pétition de principe, pour nous [la CCI] c'est au contraire une conclusion. Après une année d'activité à la Chambre de Commerce et d'Industrie, une année de contacts divers et variés, tant au niveau de Bordeaux, Pau, Toulouse ou Paris, au cours desquels nous avons rencontré un nombre incalculable de difficultés de toute sorte, nous en arrivons à conclure, sur un plan strictement économique, pour assurer un meilleur avenir économique à cette région, qu'il faudrait que Bayonne soit préfecture, pour pouvoir prendre un développement plus grand »(Journal Sud-Ouest, 4/03/1975). Cependant, à partir de 1976, la CCI va prendre en compte dans son projet la dimension identitaire (on peut citer ici une publication de la CCI de février 1976 : « la création d'un nouveau département doit être intimement associée à la défense et à la promotion de

\footnotetext{
${ }^{6}$ A titre d'exemple, le mouvement Enbata n'a réuni que 2,7\% des voix aux élections cantonales de 1967.
} 
toute culture vernaculaire $»^{7}$ afin de renforcer sa cause en lui adjoignant de nouveaux alliés : «ce phénomène traduit $[\ldots]$ un raccordement de réseaux. En participant (en suscitant) à la création de l'Association pour un Nouveau Département, la Chambre de Commerce et d'Industrie a choisi, délibérément ou non, l'ambivalence et la complexité : il fallait faire de cette revendication un projet local aussi activé que possible, en sachant, a priori, que l'hostilité de la majorité des élus dominants et du préfet était acquise. Il y a manifestement eu prise de risques car le jeu n'était pas possible sans l'appui au moins d'une partie de la sensibilité nationaliste » (Chaussier, 1996 : 84). Cependant, cette «prise de risque » ne débouchera pas sur la création d'une idéologie nouvelle, qui aurait relié revendications économiques et identité dans une même grille de lecture et d'évaluation du réel: au sein de l'AND, « on en reste à une juxtaposition des deux composantes théoriques, comme on en restait à une juxtaposition des deux formations sociales correspondantes. C'est le problème de la synthèse introuvable, de la difficulté à penser la culture comme substance essentielle du développement économique, non comme un simple environnement de type ornemental »(Chaussier, 1996: 128). De la même manière, à la fin des années 1970 et au début des années 1980, le PS va alimenter son entreprise politique d'implantation en Pays basque, terre traditionnellement de droite, sur les réseaux autonomistes qu'il va mobiliser par des alliances électorales locales (alliance avec HAS-EHAS, parti socialiste basque, lors des législatives de 1978) et par la reprise au niveau national de la revendication départementale dans les propositions du candidat F. Mitterrand en $1981^{8}$ (Chaussier, 1996).

Pour autant, la reprise de la revendication départementaliste par des groupes extérieurs à la mouvance autonomiste n'a pas débouché sur sa concrétisation. Celle-ci s'est heurtée, d'une part, à l'opposition de la majorité, toujours massivement à droite, des élus locaux, effrayés par la dimension séparatiste du projet, et plus particulièrement à celle des élus dominants (députés, sénateurs, maires de grandes villes, conseillers généraux) qui ne veulent pas remettre en cause les relations d'échanges de type «notabiliaire » (Crozier, Friedberg, 1977) qu'ils ont nouées au sein du système

\footnotetext{
7 Activités en Pays basque, n² 280, février 1976 (supplément intitulé «Un nouveau département, pourquoi ?»)

${ }^{8}$ Aujourd'hui, la reprise de la thématique départementale par le parti écologiste (les Verts) traduit le même souci de trouver un ancrage et des soutiens locaux.
} 
départemental. D'autre part, les pressions diplomatiques de l'État espagnol, qui refuse tout ce qui pourrait être interprété comme un signe positif en faveur du nationalisme basque, ont également contribué à faire reculer le gouvernement socialiste français après 1981.

La «perspective » (Schütz,1998) départementaliste, qu'elle s'inscrive dans un horizon autonomiste ou pas (pour les milieux économistes ou le parti socialiste) a donc débouché, au début de années 1980, sur une impasse du fait de l'opposition d'acteurs et d'institutions qui ont su et ont eu la capacité d'imposer leurs propres perspectives, favorables à un maintien du statu quo situationnel. La mobilisation s'est alors essoufflée et a, peu à peu, disparu au cours des années 1980, de l'agenda public national puis local.

Cependant, si la fin de non-recevoir adressée à la revendication institutionnelle constitue évidemment un problème pour les groupes autonomistes qui la défendaient, elle en constitue également un dans la perspective radicalement différente de l'État français, et plus particulièrement de son représentant local : le sous-préfet de Bayonne en poste au début des années 1990. En effet, le refus de l'État de toute concession institutionnelle, notamment sous les pressions locales et nationales, place ce sous-préfet dans une situation problématique : il doit faire face à ce qu'il perçoit, de son point de vue, comme un risque, celui de voir le mouvement autonomiste se radicaliser, i.e. opter, faute de succès probant par la voie des urnes ${ }^{9}$ et faute de perspective d'avancer dans la négociation avec l'État, pour le recours à la force. La perception de ce risque est alimentée et entretenue également par le climat de violence qui règne côté espagnol (qui fait figure ici d'exemple « repoussoir ») et la peur de voir ETA « exporter» ses pratiques violentes de ce côté des Pyrénées, alors même que l'activité d'Iparretarak en France est très réduite. Le problème pour ce sous-préfet se pose en des termes induits par l'exécution de son rôle social : ceux du maintien de l'ordre public dont il a la charge. Et c'est précisément dans cette perspective que le cadre du

\footnotetext{
${ }^{9}$ Même si depuis le milieu des années 1980 et la reconstitution d'une scène politique nationaliste en Pays basque Nord, l'impact électoral du mouvement autonomiste est en perpétuelle progression (17\% pour l'ensemble des partis au cantonales de 2001), il reste largement minoritaire, ce qui induit un nombre de postes d'élus réduits dans un système électoral qui privilégie, dans l'ensemble, le vote majoritaire par rapport au vote proportionnel. La situation est à ce titre radicalement différente du Pays basque espagnol où la majorité des électeurs votent pour des partis nationalistes.
} 
développement territorial va lui apparaître comme une opportunité cognitive à saisir pour répondre au problème porté par les autonomistes (la reconnaissance de l'identité basque) tout en le redéfinissant comme un problème de développement, évacuant ainsi la dimension institutionnelle sur laquelle il ne peut pas céder. Cependant, comme nous le verrons en dernier lieu, cette entreprise de pacification de la lutte politique et de l'espace public, qui visait à établir un cadre coopératif entre système politique et milieux autonomistes en lieu et place d'un cadre conflictuel, a généré des conséquences et suscité des usages que son entrepreneur n'avait pas anticipés. En particulier, elle a permis la relance et, simultanément, la reconfiguration de la cause départementaliste autour d'une argumentation consensuelle et synthétique, articulant l'identité et l'économie.

\section{Mobilisation du cadre partenarial et traduction de la thématique identitaire}

Le travail de «médiation » (Muller, 2000a) opéré par les experts dans la sphère des politiques publiques européennes comme françaises a progressivement introduit la notion de «développement local» dans le répertoire légitime des problèmes publics ou des «motifs » d'action publique. Autrement dit, cette notion fait désormais partie des catégories générales du langage politique qui permettent de définir ou de redéfinir ce qui fait problème dans une interaction concrète (Mills, 1940 ; Trom, 1999, 2001). Sont accrochées à ce motif les normes procédurales d'une grammaire situationnelle avec ces répertoires d'action (l'animation, la recherche de participation, la médiation, la quête du consensus par le débat, l'ouverture), de rôle (animateur, partenaire, consultant extérieur), de dispositifs (diagnostics territoriaux, projets, partenariat élu/expert/administration/société civile, contrat, évaluation participative). Ce motif et ces normes définissent ensemble un langage de l'action constitutif, ce que nous avons appelé le cadre du développement territorial.

$\mathrm{Du}$ point de vue du sous-préfet, ce cadre constitue une ressource publiquement disponible, cautionnée par l'autorité experte, qu'il mobilise 
dans une «performance ${ }^{10} »($ Trom, 2001) de redéfinition de la situation qui vise des effets situés. À travers ou par cette performance, l'État visait à transformer le cadre cognitif et normatif dans lequel les autonomistes percevaient leur relation à l'État et aux autorités politiques locales: il s'agissait de passer d'un cadre de type conflictuel à un partenariat ou une concertation, de transformer la contestation de l'ordre politique en mobilisation autour d'une action publique afin de prévenir toute évolution favorable à l'instauration d'un climat de violence politique. Comme le reconnaît sans ambages un élu, acteur et partisan de la démarche depuis son origine : «les arrières pensées politiques existaient [...] incontestablement: celles et ceux qui ont lancé cette opération cherchaient peut-être, certainement même, à construire un projet au Pays basque pour éviter les dérives $\gg^{11}$. Le cadre du développement fournit la base symbolique de cette opération : il permet à la fois de reconnaître la légitimité de la parole autonomiste tout en la filtrant et en la traduisant de sorte que la thématique de l'identité se trouve déplacée du registre conflictuel de la lutte pour la reconnaissance politique (politics) à celui, pacifié, de l'action publique concertée (policy). Pour le dire autrement, dans la perspective du sous-préfet, le cadre du développement local se révèle pertinent en ce qu'il lui paraît de nature à l'aider à gérer ce qu'il considère comme un risque et ainsi exécuter une des missions définies par son rôle social (maintenir l'ordre public).

Le premier objectif du sous-préfet, en lançant la démarche Pays basque 2010 en 1992, est de rompre avec une situation où la parole autonomiste ne trouvait pas ou peu écho et de relais dans le système politique et vers l'action publique : "au début des années 1990, à l'initiative du souspréfet de l'époque s'est engagée une réflexion qu'on appelait Pays basque 2010, puis Schéma de Développement du Pays basque [...]. Il avait constaté qu'au Pays basque il y avait une espèce de schizophrénie entre les élus du peuple [...] qui géraient les collectivités locales, participaient aux négociations de dossier avec l'État et puis les autonomistes qui eux, compte tenu du fait qu'ils n'avaient jamais eu un impact électoral considérable n'étaient pas élus mais occupait en grande partie le débat public, les médias,

\footnotetext{
${ }^{10}$ La notion de performance, qui induit la mobilisation d'une grammaire, souligne également l'importance de la médiation de l'acteur entre la situation et le cadre mobilisé pour la (re)définir (Blumer, 1969).

${ }^{11}$ Extrait d'un entretien réalisé avec un élu, membre du CD et du CE, le 11/12/2000.
} 
les agitations, et les manifestations et lançaient des idées parfois intéressantes et que ces deux mondes-là s'ignoraient. Alors il a proposé de manière tout à fait opportune que tout le monde se mette autour de la table pour essayer de réfléchir ensemble à l'avenir du Pays basque ${ }^{12}$. Cette situation, nous l'avons $\mathrm{vu}$, apparaît dans la perspective du sous-préfet comme potentiellement «dangereuse» en ce qu'elle risque d'encourager des positionnements « extrémistes ». Plutôt que de simplement surveiller cette situation, il a alors tenté de la recomposer en changeant la perception des différents protagonistes (élus, administratifs, mouvement autonomistes) sur la situation, i.e. sur ce qu'ils peuvent ou doivent y faire et les relations qu'ils peuvent y entretenir. Ce sous-préfet, qui s'inscrit dans la tradition française des sous-préfets animateurs, a alors mobilisé, avec l'appui d'experts du développement qu'il engage pour encadrer la démarche, le cadre du développement qui lui permet de proposer aux élus locaux et aux autonomistes une grille d'interprétation $\grave{a}$ même de modifier profondément leur perception de la situation et, en particulier, de dépasser le conflit qui les oppose. Il s'agit donc d'une performance expressive, au sens où elle vise à influencer l'interprétation (opération cognitive) que les autres interactants font de la situation (De Queiroz, Ziolkowski, 1997).

Le cadre du développement a ainsi jeté les bases symboliques d'un changement dans la perception de la relation élus/autonomistes : ceux-ci ne s'identifient plus uniquement comme des adversaires engagés dans une lutte politique mais également comme des partenaires engagés dans un jeu à somme non nulle. L'idéologie du développement fournit ainsi un nouveau répertoire de rôles inscrit dans une relation de complémentarité et de reconnaissance réciproque : pour les élus, celui d'animateur/médiateur du débat, qui associe et mobilise les compétences, pour les autonomistes celui de partenaire social, qui contribue à relayer les attentes du territoire et ses besoins. La démarche est donc d'abord le vecteur d'un apprentissage de nouvelles façons de faire et de nouvelles manières de considérer l'autre: «Moi comme jeune élu, car ça fait huit ans que la démarche est lancée, j’ai beaucoup appris sur la manière peut-être nouvelle de faire de la politique, c'est-à-dire avant tout de mobiliser des acteurs de territoire qui d'ailleurs peuvent avoir vis-à-vis du politique incontestablement une certaine défiance et qui là se retrouvent dans des séances de travail, loin des effets de tribune et

\footnotetext{
${ }^{12}$ Extrait d'un entretien réalisé avec un élu, membre du CE et du CD, le 2/02/2001.
} 
de tout ce qui peut aujourd'hui entraîner un effet repoussoir à la politique, et parallèlement pour les élus, c'est certainement très enrichissant que d'arriver à mobiliser tant de compétence pour construire un projet de territoire ». Plus loin dans l'entretien, cet élu se félicitera de «l'apparition d'un dialogue entre élus, comme moi RPR, et mouvement associatif abertzale ${ }^{13}$ inimaginable il y a 10 ans : l'image des élus de droite a changé dans le milieu associatif et réciproquement $»^{14}$. Le succès que va rencontrer localement cette grammaire relationnelle s'explique par l'intérêt qu'elle suscite auprès de ces deux groupes : elle fournit d'une part une base cognitive et normative aux élus pour redéfinir leur rôle et leur légitimité dans un contexte où celle-ci est fortement contestée, alors qu'elle offre une opportunité aux autonomistes pour changer d'image (apparaitre comme des partenaires responsables plutôt que comme des terroristes potentiels) et faire avancer certaines de leurs idées, concernant notamment la défense et la promotion de la culture basque.

Il est important ici de noter que la redéfinition de la relation administration/élus/autonomistes s'appuie non seulement sur des discours et l'affichage de bonnes intentions mais sur des dispositifs et des épreuves (Boltanski, Thévenot, 1991) empruntés au cadre du développement qui confèrent une crédibilité générale à l'activité dans laquelle les interactants sont engagés (à ce titre, il a pu être jugé à une époque comme « exemplaire » par la DATAR). Ainsi, l'agencement de la situation au cours du temps par les promoteurs de la démarche traduit leur volonté d'objectiver la participation à la fois par des dispositifs de consultation (Conseil des développements, Conseil des Élus) qui rendent le débat visible et par des documents qui attestent de l'engagement réciproque des «partenaires» (schéma de développement, convention spécifique) vis-à-vis d'un projet d'action consensuel. L'objectif des initiateurs de la démarche ne consistait pas simplement à afficher une « ouverture » de façade mais bien à «instituer » un cadre d'échange politique susceptible d'échapper à toute critique, un cadre légitime (Douglas, 1999). De fait, si cette politique était symbolique, au sens où elle visait à agir sur les représentations du réel, elle a eu des conséquences très concrètes sur l'action publique: en particulier, la participation autonomiste à la consultation au sein du Conseil de Développement s'est traduit par la forte inflexion culturelle donnée au Schéma d'Aménagement

\footnotetext{
${ }^{13}$ Abertzale signifie en basque «patriote » et désigne les partisans de la cause autonomiste.

${ }^{14}$ Extrait d'un entretien réalisé avec un élu, membre du CE et du CD, le 11/12/2000.
} 
pour le Pays basque achevé en 1997, avec par exemple le développement d'une politique de soutien linguistique pour la langue basque.

Cependant, à travers l'institution de ce cadre, l'État poursuit une stratégie qui vise à «éviter les dérives », i.e. à réguler la contestation de l'ordre politique en offrant aux contestataires une opportunité d'investissement dans la construction de l'action publique. Il s'agissait pour l'État français de proposer aux autonomistes une alternative crédible à la «défection » (exit) vis-à-vis du système politique, en ménageant la possibilité d'une « prise de parole » (voice) à l'intérieur de ce système dans le cadre d'un projet d'action publique (Hirschman, 1995). L'objectif était alors «d'institutionnaliser » la contestation, c'est-à-dire de la décharger de sa composante conflictuelle et polémique (en particulier la revendication d'un institution politique basque) en lui donnant un cadre susceptible de la transformer en participation et ainsi renforcer la légitimité du système politique dans son ensemble en créant de la «loyalty » pour les institutions politiques en place chez les autonomistes (Hirschman, 1995). Le cadre est alors le support cognitif de cette entreprise «d'émasculation» de la contestation (Hirschman, 1995).

De fait, la démarche Pays basque dote l'expression autonomiste d'un cadre (celui du développement) où elle est reconnue. Mais, ce cadre, en même temps qu'il habilite une médiation vers l'action publique, contraint la mise en forme de la parole autonomiste en traçant des frontières cognitives et normatives à son expression. La parole autonomiste s'en trouve à la fois banalisée, filtrée et in fine traduite ${ }^{15}$ (Callon, 1996 ; Lascoumes, 1994) dans un langage nouveau.

Le cadre du développement induit tout d'abord une banalisation du mouvement autonomiste. Ainsi, si des représentants du monde culturel et associatif, autonomistes ou proches de l'autonomisme, se voient reconnus en tant que «partenaires", c'est au même titre et sur le même plan que les représentants du monde économique. De fait, les promoteurs de la démarche consultative visent non seulement les autonomistes mais « toute la population, disons les forces vives du Pays basque, qui comprennent à la fois des milieux

\footnotetext{
${ }^{15}$ Par traduction, nous entendons un déplacement de sens d'un répertoire social à un autre, ou d'une grammaire sociale à une autre, afin d'intéresser des acteurs à une action ou de discréditer un adversaire.
} 
culturels, associatifs mais aussi des milieux économiques notamment la Chambre de Commerce et d'Industrie de Bayonne ${ }^{16}$. En effet, la démarche entend aussi répondre aux attentes «en médiation» des milieux économiques qui trouvent que les institutions politiques actuelles représentent mal leurs intérêts. De fait, au cours de la procédure consultative, les valeurs identitaires portées par les autonomistes vont devoir composer avec les intérêts des acteurs économiques : la singularité de la revendication identitaire se trouve alors diluée dans un «consensus de composition» (Boudon, Bourricaud, 1982) où l'identité n'est ni plus ni moins légitime que l'économie.

De plus, la dimension politique des revendications autonomistes est évacuée: les promoteurs de la démarche laissent soigneusement les revendications institutionnelles des autonomistes hors cadre (filtrage de la parole). Il s'agissait alors de s'engager dans un « débat tout à fait raisonnable autour de la question économique, sociale et même culturelle et de valeurs qui pouvaient être résolue avec tout le monde, qui était un débat où tout le monde pouvait être engagé alors que le département nous aurait engagés dans un autre débat où il $\mathrm{y}$ aurait eu des fractures entre les uns et les autres ${ }^{17}$. Le contournement de la question institutionnelle est donc ici légitimé au nom de la recherche du dialogue et du consensus qui font figure de norme dans la grammaire du développement local. Dans ce cadre, la question de l'institution s'efface devant celle de l'action publique.

Enfin, si la parole identitaire est prise en compte dans le cadre, c'est au prix d'une traduction qui fait de l'identité une thématique consensuelle. Les valeurs identitaires portées par les autonomistes se trouvent ainsi transcodées dans le langage du développement qui permet de les redéfinir, en particulier via le travail des experts qui construisent ces représentations nouvelles et les dotent d'une autorité ${ }^{18}$ (Boudon, 1986). L'identité, à travers ce travail, est redéfinie comme une ressource mobilisée dans une démarche de développement. Ainsi le sentiment d'appartenance n'est pas interprété, comme dans les idéologies identitaires, comme ce qui fonde la contestation de l'ordre politique, mais comme un élément qui permet de fédérer les énergies derrière le projet, de susciter des rapprochements, comme un «moteur»

\footnotetext{
${ }^{16}$ Extrait d'un entretien réalisé avec un élu, membre du CE et du CD, le 29/01/2001.

${ }^{17}$ Extrait d'un entretien réalisé avec un élu, membre du CE et du CD, le 10/12/2001.

${ }_{18} \mathrm{R}$. Boudon parle d'effet d'autorité quand on accepte le point de vue d'une personne socialement considérée comme compétente dans le domaine considéré (Boudon, 1986).
} 
humain du développement. L'identité devient alors un facteur d'engagement dans la démarche sur lequel celle-ci doit s'appuyer: «ce que nous avons essayé de faire au niveau du Pays basque, c'est bien entendu travailler sur une communauté de projet et une communauté de destin auxquelles il fallait superposer un attachement linguistique et culturel encore beaucoup plus évident que dans d'autres régions, ce qui fait que le territoire sur lequel nous avons travaillé présentait des particularités [...] sur lesquelles on doit s'appuyer si on veut faire bouger les choses [...]. La notion de développement n'est pas une notion que l'on peut plaquer, ce n'est pas une notion qui peut se développer à partir de directives, c'est une notion qui ne peut se développer que s'il y a un sentiment d'appartenance à quelque chose, un facteur humain..., la possibilité pour les hommes de mouiller leur maillot [...]. Il faut du sentiment d'appartenance, il faut du volontarisme de la part des élus et des principaux acteurs, de tous les acteurs [...] $\gg^{19}$. L'identité ne constitue pas dans ce cadre normatif et cognitif une fin en soi mais un médium dans une stratégie globale d'animation du territoire dont l'objectif est le développement du Pays basque. De fait, dans cette perspective, l'identité ne doit pas être un prétexte à un « repli sur soi » ou sur la tradition qui irait contre les normes d'ouverture et de changement que porte le cadre du développement: «c'est un pays [un territoire de projet] qui correspond à la volonté de ses habitants, de ces élus de travailler ensemble, de créer quelque chose ensemble. L'identité pour moi c'est ça, c'est pas brandir un fusil, c'est se reconnaître comme appartenant à une histoire, à un vécu, à une langue, à des coutumes [...]. Il me semble qu'est venu le moment de se ressourcer, pas de se recroqueviller, de se ressourcer sur ses bases, sur ses racines, sur son histoire, sur son identité pour proposer des choses, pour faire des projets ensemble, etc. mais pas encore une fois pour se recroqueviller, non, en même temps être plus fort sur place et dans l'environnement de l'Europe qui est un enjeu considérable aujourd'hui pour l'avenir. Le «pays» n'est pas une fermeture mais une ouverture $»^{20}$. L'identité est ici lue non sur le mode contestataire du séparatisme et de l'engagement dans la lutte pour la reconnaissance mais sur celui du rapprochement (l'identité fédératrice) dans l'action publique et de l'ouverture au monde, à la modernité, aux partenaires extérieurs (dont l'État). Cette nouvelle mise en intrigue de l'identité basque dans un récit d'action publique

\footnotetext{
${ }^{19}$ Extrait d'un entretien réalisé le 13/01/2001 avec un élu, membre du CE et du CD.

${ }^{20}$ Extrait d'un entretien réalisé avec un élu, membre du CE et du CD, le 4/01/2001.
} 
(policy narrative) qui mobilise le langage du développement trouve son expression la plus nette dans ce qui constitue le «texte de référence » de la démarche : le projet de territoire Lurraldea. Ce projet se structure autour de quatre enjeux dont «l'alliance de la culture et de la modernité » qui suppose de «prendre appui sur le sentiment d'appartenance à une communauté de destin pour : 1) revaloriser l'identité ; 2) gérer le Pays basque comme un bien collectif et indivisible ; 3 ) provoquer l'échange entre culture basque et culture universelle ; 4) s'affirmer comme terre d'ancrage et de modernité ».

Le nouveau cadre, qui mobilise la grammaire du développement, induit donc une nouvelle représentation de l'identité et du territoire (non plus le territoire de la communauté mais le support d'un dialogue et l'objet d'une action publique) qui se pose en alternative consensuelle aux représentations conflictuelles des idéologies identitaires. La culture et la singularité basque sont à la fois reconnues et profondément requalifiées, ou pour être plus précis retraduites de telle manière qu'elles n'apparaissent plus comme un objet de conflit mais comme le support d'une action publique.

\section{L'appropriation autonomiste du cadre partenarial}

L'opération symbolique entreprise par l'État, avec l'aide d'experts, visait donc des milieux qui contestent l'ordre établi et revendiquent un changement institutionnel: d'une part, et en priorité les mouvements autonomistes, chez qui la création d'un département basque est une vieille revendication, mais également le milieu économique local qui s'est déjà par le passé montré favorable à la création d'un nouveau département. L'objectif est de proposer à ses mouvements un nouveau cadre dans lequel les problèmes locaux sont formulés non pas en terme d'institution (un problème de représentation politique) mais de dialogue et de projet d'action (un problème d'action publique). Pour autant les mouvements visés par ce recadrage n'ont pas accepté cette nouvelle représentation de la réalité telle quelle : ils ont au contraire réinterprété ce cadre dans la perspective qui est leur est propre, à savoir celle de la lutte pour une reconnaissance institutionnelle du territoire basque. Ils se sont ainsi livrés à une opération de « contre-cadrage » (Benford, Hunt, 2001) qui leur a permis à la fois de redéfinir leur position de contestation (i.e. de trouver de nouveaux arguments, de nouvelles justifications pour leur cause) et de mobiliser de nouveaux soutiens. 
Cette opération symbolique de contre-cadrage a impliqué à la fois la mouvance autonomiste et les représentants du monde économique. En effet, alors que l'AND s'était contentée au tournant des années 1970 et 1980 de juxtaposer les deux revendications et ce au prix de tensions internes très fortes (Chaussier, 1996), la «démarche de projet» a raccordé plus fortement les réseaux nationalistes et économiques en leur fournissant une base cognitive permettant de concilier lutte pour la reconnaissance et défense des intérêts locaux. Le pont sémantique jeté par les experts entre identité et développement a ainsi permis la constitution d'un terrain d'entente symbolique intégrant les deux formes de revendication départementaliste. La question de la reconnaissance a pu ainsi être reprise par certains représentants des milieux économiques "parce qu'un département concrétiserait l'identité Pays basque et il y a une corrélation forte entre sens de l'identité et développement. Le plus souvent identité égale développement : la création de cette institution serait un choc psychologique qui permettrait un élan collectif de développement. Donc il faut reconnaître l'identité Pays basque et il faut la reconnaître par une institution $»^{21}$. L'association reconnaissance/développement fonctionne alors comme le «ciment symbolique » qui permet la réunion des deux réseaux: elle constitue «l'oriflamme publique [...] sous laquelle tous peuvent travailler de concert » car ils ont " l'assurance que sur ce point au moins, tout le monde est d'accord» (Strauss, 1992 : 95). Bien entendu, ce «ciment symbolique » masque des désaccords et des divergences d'objectifs considérables entre autonomistes et milieux économiques (en particulier, les milieux économiques ne voient pas dans le département, contrairement aux autonomistes, une étape vers l'indépendance à laquelle ils sont majoritairement opposés) mais il constitue tout de même un «mandat généralisé » qui permet la constitution d'une mobilisation unifiée (Strauss, 1992).

Les deux réseaux, rapprochés par la démarche, l'ont alors saisie pour relancer et redéfinir la revendication départementaliste : il ont détourné le cadre d'action publique qui leur était proposé pour lui donner un sens polémique et le raccorder à leur lutte politique. Nous désignerons cette opération de contre-cadrage, ou de transformation du cadre, par le terme de

${ }^{21}$ Extrait d'un entretien réalisé le 19/12/2000 avec un membre du Conseil de Développement, proche des milieux d'affaires locaux (directeur de Banque en retraite) et pour qui la priorité est de « contribuer au développement économique » du territoire. 
«modalisation» (Benford, Hunt, 2001). Chez Goffman un mode désigne «l'ensemble des conventions par lesquelles une activité donnée, déjà porteuse de sens dans un cadre primaire, est transformée en quelque chose qui se modèle sur cette activité mais qui est vu par les participants comme radicalement autre (Goffman, $1991: 52$ ). R. Benford et S. Hunt proposent d'appliquer ce concept «dans le champ des mouvements sociaux » où « la modalisation advient quand les participants d'un mouvement reformulent les propositions de leurs opposants en en neutralisant, subvertissant ou retournant le sens. La modalisation est un processus dialectique de réattribution, de réinterprétation et de recodage du sens - une activité de recadrage » (Benford, Hunt, 2001: 179). Ainsi, les départementalistes proposent une nouvelle lecture de la démarche qui altère profondément le sens que lui donne ses promoteurs. De fait, ils font de la construction du Schéma d'Aménagement un argument au service de leur cause : «le projet de département Pays basque a réapparu en 1996 après 20 ans de sommeil à la suite de l'opération «Pays basque 2010 » qui a abouti au Schéma de Développement. Tous les gens qui avaient participé à cette démarche, c'est-à-dire les forces vives du Pays basque [...] se sont aperçus que tout ça n'avait pas de $\operatorname{sens}^{22}$ s'il n'y avait pas une institution, une véritable institution avec des moyens humains et des moyens financiers, avec une représentativité, avec une personnalité morale qui concrétise l'identité Pays basque et soit un vecteur de cet élan de développement $\gg^{23}$

Nous sommes bien ici en présence d'une modalisation: le schéma n'est ni ignoré ni critiqué ${ }^{24}$ mais son sens est réinterprété dans un nouveau cadre où il devient un appui pour la revendication départementale, appui d'autant moins contestable que sa validité et son exemplarité ont été reconnues par l'État, la DATAR, et les élus des partis traditionnels. Les départementalistes réinvestissent la légitimité du projet, acquise dans le domaine de l'action publique, dans leur lutte. Le département est désormais revendiqué au nom de la topique consensuelle du développement, qui allie

${ }^{22}$ C'est nous qui soulignons.

${ }^{23}$ Extrait d'un entretien réalisé le 19/12/2000 avec un membre départementaliste du Conseil de Développement.

${ }^{24} \mathrm{R}$. Benford et $\mathrm{S}$. Hunt dressent une véritable typologie des réponses des mouvements sociaux aux cadres que leur opposent (ou proposent) leurs adversaires : s'ils peuvent les «modaliser », ils peuvent également les «ignorer», les «endosser », y «riposter» ou s'en «démarquer» (Benford, Hunt, 2001). 
intérêt économique et identité. Cette institution est alors justifiée en tant qu'elle apporterait un "poids politique», des moyens «financiers et techniques » au projet et serait responsable «vis-à-vis de ses électeurs » de sa bonne marche.

Parallèlement, les départementalistes discréditent (Goffman, 1991) le cadre non transformé que leur proposent l'État et les grands élus. Le contrecadrage jette ainsi un doute sur la capacité du territoire de projet à déboucher sur une action publique : «si le territoire de projet est un outil de réflexion il n'est en aucun cas un moyen d'action. Il n'a pas de personnalité morale. Il n'a aucune représentativité alors qu'un conseil général est élu. Donc il n'a pas de responsabilité vis-à-vis des électeurs. Il n'a aucun moyen financier par conséquent il ne peut pas mettre de l'argent lui-même dans le projet. Et enfin, [il] n'a aucun moyen humain [...]. S'il n'y a pas une institution avec des moyens humains, de services techniques et autres pour piloter, contrôler, impulser, veiller à l'avancement des actions, etc., jamais il n'y aura une réalisation complète ou aussi rapide qu'il serait souhaitable du Schéma de Développement ; c'est une constante de la vie, s'il n'y pas de responsables quelque part, les choses n'avancent pas bien. Seul le département, une institution Pays basque pourrait être responsable $»^{25}$. Le discrédit prend aussi parfois la forme du soupçon qui pointe le cadre comme l'instrument d'une machination ou d'une «fabrication » (Goffman, 1991) : le territoire de projet, sans l'appui d'un institution basque, est alors dénoncé comme un «leurre ${ }^{26}$ destiné à « détourner les gens de leur but qui est la création d'une institution Pays basque $»^{27}$.

Le contre-cadrage opéré par les départementalistes fait donc du schéma de développement un élément d'un argumentaire anti-« ordre établi », alors qu'il a été précisément construit pour re-légitimer cet ordre. Cet argumentaire va rencontrer un vif succès auprès des élus locaux à tel point

\footnotetext{
${ }^{25}$ Extrait d'un entretien réalisé le 19/12/2000 avec un membre départementaliste du Conseil de Développement.

${ }^{26}$ Le même soupçon est entretenu par les départementalistes au sujet de la convention de financement du projet signé en 2001 : la convention n'engagerait pas vraiment l'État ( en dernière analyse c'est l'État qui fait les choix finaux », qui «distribue les crédits ») face à un partenaire (le territoire de projet) qui n'aurait pas de poids juridique et politique, et donc ne garantirait pas la mise en œuvre du projet.

${ }^{27}$ Extrait d'un entretien réalisé le 4/05/2001 avec un élu autonomiste.
} 
qu'une nette majorité des maires va se prononcer en faveur de la création d'un département «Pays basque » lors d'une consultation organisée en 1996 par l'Association des Élus pour un Département basque. Ce succès tient, à notre avis, à la fois au recadrage des motifs de lutte et au cours suivi par la démarche. En effet, la formulation d'un motif doit être analysée, non pas simplement comme l'explicitation de la raison de l'action, mais comme un acte linguistique qui produit des effets sur l'action collective : ainsi, le choix d'un motif approprié dans le «vocabulaire des motifs » socialement légitimes peut permettre de gagner de nouveaux soutiens à une cause en l'inscrivant dans un horizon de justification ${ }^{28}$ plus «porteur» (Mills, 1940). Dans le cas qui nous occupe, la motivation de la mobilisation pro-départementale se trouve profondément reformulée: ce n'est plus la poursuite d'un idéal autonomiste qui est mise en avant mais la mise en place d'un programme d'action consensuel qui vise le développement du territoire. La mobilisation du motif «développement local» a facilité le ralliement des milieux économiques et d'une grande partie des élus locaux auparavant effrayés par la dimension «séparatiste » du projet départemental. Cependant, si ces acteurs se retrouvent dans cette revendication, c'est non seulement parce qu'elle prend appui sur un projet consensuel mais également parce que le cours de l'interaction renforce l'argumentation des départementalistes : la lenteur et les difficultés dans la mise en œuvre du projet (il faudra attendre fin 2000 pour qu'une convention de financement soit signée pour un projet finalisé en 1997) ont renforcé la crédibilité des dénonciations émanant des départementalistes.

La démarche « Pays basque 2010 » qui visait à institutionnaliser et à neutraliser la contestation a donc eu précisément l'effet inverse : la démarche est l'un des principaux vecteurs et supports de la relance de la revendication départementaliste au milieu des années 1990. Le rapprochement espéré par l'État entre élus et société civile s'est bien opéré, mais dans un sens contraire à celui qui était voulu par les promoteurs de la démarche : la contestation de

\footnotetext{
${ }^{28}$ Ceci ne signifie évidemment pas que les motifs n'ont aucun rapport avec l'action réelle dont il ne serait que l'habillage légitimant : de fait les conventions sociales sur les motifs légitimes contribuent à définir l'éventail des conduites possibles en contexte. Ainsi, comme le montre bien C. W. Mills, le vocabulaire des motifs acceptables socialement dans une situation donnée contraint bien souvent l'action : « to term [motives] justifications is not to deny their efficacy. Often anticipations of acceptable justifications will control, conduct. ( If I did this, what could I say? What would they say?»). Decision may be, wholly or in part, delimited bay answers to such queries. » [Mills, 1940, p. 907].
} 
l'ordre institutionnel a gagné une grande partie des élus. Seuls, les «grands élus » (députés, sénateurs, maires de grandes villes, conseillers généraux), qui entretiennent avec ou dans les institutions en place, hostiles à tout changement (l'État central, la préfecture, le Conseil général), des relations d'échange privilégiées de type notabiliaire (Crozier, Friedberg, 1977), restent dans leur grande majorité favorable au statu quo.

On aurait donc tort de considérer les autonomistes comme les victimes d'un «coup de force symbolique » (Bourdieu, 1987) qui verrait l'État leur imposer, en s'appuyant sur l'autorité des experts, une nouvelle définition de la situation qui mettrait l'accent sur la participation et l'action publique. Au contraire, les autonomistes, s'ils ont accepté le nouveau cadre, se le sont appropriés : ils l'ont modalisé, réinvestissant la forme "projet » d'un sens conflictuel et polémique. Leur appropriation du cadre a débouché sur une stratégie d'action complexe qui joue à la fois sur les registres sociaux de la participation (ou du dialogue social) et de la mobilisation (ou du conflit social).

En effet, d'un côté, le mouvement autonomiste a joué le jeu du dialogue social, de la négociation autour de l'action publique. En participant à l'élaboration du Schéma d'Aménagement (1992-1997), les autonomistes ont investi la «policy window» (Kingdon, 1984) ouverte par «Pays basque 2010 », fenêtre qui leur offrait l'opportunité d'obtenir à la fois un engagement de l'État et des collectivités sur des actions de défense culturelle et linguistique et une forme de reconnaissance du territoire. Le projet une fois élaboré, les autonomistes ont également profité, avec leurs alliés du monde économique, des possibilités de "prise de parole» qu'offre le cadre participatif pour diffuser et exprimer une parole critique. Ainsi, le Conseil de Développement, chargé à partir de 1998 de l'évaluation de la mise en œuvre $\mathrm{du}$ projet, va devenir un lieu d'expression de la revendication départementaliste (lors d'un vote organisé en 1999, ce Conseil va même se prononcer officiellement pour la création d'un département). Les outils de la participation se trouvent alors retournés contre ceux qui pensaient pouvoir les utiliser pour évacuer la question institutionnelle (l'État, les grands élus). De plus, en refusant le débat sur la question institutionnelle à de multiples reprises entre 1996 et 2001, le Conseil des Élus, contrôlé par les grands élus opposés au projet départemental, va s'exposer à des critiques qui mobilisent 
certains des principes qui sont au cœur de la légitimation de la démarche (l'ouverture, le dialogue).

De l'autre côté, les autonomistes ont pris appui sur le projet pour relancer une mobilisation sociale. Ainsi, la démarche de projet est également saisie par les autonomistes comme une base normative et cognitive qui permet de redéfinir la cause départementale et de lui adjoindre de nouveaux soutiens (alliés économiques, «petits » élus). Ces soutiens économiques et politiques, gagnés par le recadrage de la cause, ont été mobilisés non seulement dans le cadre du débat public institutionnalisé mais également dans le cadre de performances relevant du registre de la mobilisation et du conflit social, et ce dès le milieu des années 1990: des manifestations, des pétitions, des consultations (comme le vote des maires) sont organisées, de sondages sont commandés, des organisations de lutte ancienne sont réactivées (l'Association pour un Nouveau Département, l'Association des Élus pour un Département Pays basque). Il s'agit alors, non pas d'invoquer les principes bafoués de la participation, mais de faire appel à la pression publique, que l'on atteste à travers des dispositifs empruntés à la "grammaire civique » (manifestation, vote, sondages, etc.), dispositifs qui présentifient le soutien collectif (ici celui des habitants du Pays basque au nom de qui la lutte est menée) afin de faire pression sur l'État et les grands élus (Boltanski, Thévenot, 1991).

\section{Conclusion}

Cet article montre comment les savoir-faire, les croyances, les normes et les valeurs attachés à la démocratie délibérative peuvent être transformés, de manière concurrentielle, en ressources politiques par des acteurs et groupes en conflit. De fait, en Pays basque, le cadre partenarial est investi par les pouvoirs publics en tant qu'il constitue une opportunité pour transformer la contestation de l'ordre politique en participation à une action publique. Cependant, l'Etat n'a pas le monopole de l'appropriation du discours «modernisateur»: nous avons ainsi souligné comment les autonomistes envisagent, transforment et s'approprient ce cadre dans la perspective de la lutte pour la reconnaissance institutionnelle du Pays basque qu'ils mènent, n'hésitant pas à jouer sur plusieurs registres d'action et de légitimation (grammaire du développement/grammaire civique) afin de renforcer leur position. Destiné à pacifier la situation et à créer de nouvelles "proximités » entre Etat et Autonomistes, le recours au registre de la participation et du 
développement a donc in fine contribué à alimenter le conflit politique identitaire en en renouvelant la forme et les enjeux.

\section{BIBLIOGRAPHIE}

Abélès, M. (1989). Jours tranquilles en 89. Odile Jacob, Paris.

Austin, J.L. (1970). Quand dire, c'est faire. Seuil, Paris.

Benford R., Hunt S. (2001). Cadrages en conflit, mouvements sociaux et problèmes sociaux. In : Cefaï D., Trom D. (Ed.). Les formes de l'action collective : mobilisation dans les arènes publiques. Ed. de l'EHESS, Paris.

Berger P., Luckmann T. (1996). La construction sociale de la réalité. Armand Colin, Paris.

Bidart P. (2001). La singularité basque. PUF, Paris.

Blumer H. (1969), Symbolic interactionism : perspective and method. University of California Press, Berkeley.

Boltanski L., Thévenot L. (1991). De la justification: les économies de la grandeur. Gallimard, Paris.

Boudon R. (1979). Effets pervers et ordre social. PUF, Paris.

Boudon R. (1986). L'idéologie ou l'origine des idées reçues. Fayard, Paris.

Boudon R., Bourricaud F. (1982). Dictionnaire critique de sociologie. PUF, Paris.

Bouilloud J-P. (1997). Sociologie et société : épistémologie de la réception. PUF, Paris.

Bourdieu P. (1987). Choses dites. Ed. de Minuit, Paris.

Callon M. (1986). «Eléments pour une sociologie de la traduction », L’Année Sociologique, p. 169-208. 
Charvolin F. (2003). L'invention de l'environnement en France : chroniques anthropologiques d'une institutionnalisation. La Découverte, Paris.

Chaussier J-D. (1996). Quel territoire pour le Pays basque? Les cartes de l'identité. Paris, L'Harmattan, Paris.

Crozier M., Friedberg E. (1977). L'acteur et le système. Seuil, Paris.

De Queiroz J.M, Ziolkowski M. (1997). L'interactionnisme symbolique. PUR, Rennes.

Dimaggio P., Powell W. (1991). The new institutionnalism in organizational analysis. University of Chicago Press, Chicago.

Douglas M. (1999). Comment pensent les institutions. La découverte, Paris.

Douillet A-C. (2003). «Les élus ruraux face à la territorialisation de l'action publique », Revue Française de Science Politique, vol. 53, n 4, p. 583-606.

Faure A. (1992). Le village et la politique, essai sur les maires ruraux en action. L'Harmattan, Paris.

Fischer F., Forester J. (1993). The argumentative turn in policy analysis and planning. Duke University Press, Durham.

Friedberg E. (1977). Le pouvoir et la règle: dynamiques de l'action organisée. Seuil, Paris.

Gaudin J-P. (1999). Gouverner par contrat : l'action publique en question. Presse de Sciences Po, Paris.

Geertz C. (1964). "Ideology as a cultural system", in : Apter D. Ideolgy and discontent. The Free Press, Glencoe.

Giddens A. (1997). La constitution de la société. PUF, Paris.

Goffman E. (1991). Les cadres de l'expérience, Ed. de Minuit, Paris.

Hirschman A. (1995). Défection et prise de parole : théorie et applications. Fayard, Paris.

Honneth A. (2000). La lutte pour la reconnaissance. Ed. du Cerf, Paris.

Joas H. (1993). Pragmatism and social theory. The University of Chicago Press, Chicago. 
Kingdon J. (1984). Agendas, alternatives and public policies. Little Brown, Boston.

Labrouche C. (s. d .). Reconstruction d'une identité territoriale à travers un projet de territoire: la convention spécifique Pays basque. Document non publié.

Lascoumes P. (1994). L'Ecopouvoir. La Découverte, (Paris).

Le Bart C. (1998). Le discours politique. PUF, Paris.

Mabileau A. (1994). Le système local en France. Montchrestien, Paris.

Mills C.W. (1940). «Situated actions and vocabularies of motive», American Sociological Review, vol. 51, p. 904-913.

Muller P. (2000a). Les politiques publiques. PUF, Paris.

Muller P. (2000b). «Vers une sociologie politique de l'action publique», Revue Française de Science Politique, vol. 50, p. 189-207.

Nay O., Smith A. (2002). Le gouvernement du compromis. Economica. Paris.

Padioleau J-G. (1982). L'État au concret. PUF, Paris.

Schütz A. (1998). Eléments de sociologie phénoménologique. L’Harmattan. Paris.

Strauss A. (1992). La trame de la négociation: sociologie qualitative et interactionnisme. L'Harmattan, Paris.

Tilly C. (1986). La France conteste de 1600 à nos jours. Fayard, Paris.

Trom D. (1999). «De la réfutation de l'effet NIMBY considéré comme une pratique militante: notes pour une approche pragmatique de l'activité revendicative », Revue Française de Science Politique, p. 31-50.

Trom D. (2001). «Grammaire de la mobilisation et vocabulaires de motifs », in: Cefaï D., Trom D. (ed.), Les formes de l'action collective : mobilisation dans les arènes publiques. Ed. de l'EHESS, Paris.

Yanow D. (1996). How does a policy mean? Georgetown University Press, Washington. 


\title{
RESUME
}

Identité en négociation :

pratiques participatives, politique de développement et conflit identitaire en Pays basque «français »

Cet article se donne pour objectif de montrer comment le cadre d'action publique (policy) investi en Pays basque autour de la notion emblématique de «développement local» a pu constituer, non seulement pour les pouvoirs publics mais également pour les groupes autonomistes, une ressource politique (politics). Cette ressource a été activée dans des stratégies de mobilisation et/ou de légitimation dans un contexte marqué par un conflit identitaire. Ce cadre participatif du développement a ainsi fait l'objet d'appropriations concurrentielles, chaque partie s'engageant dans une compétition pour l'imposition du sens légitime à donner à l'action conduite.

\begin{abstract}
Identity under negotiation :

participative practices, politics of development and identity conflict in "french" basque country

This article aims at demonstrating how the policy implemented in Basque Country about de emblematic notion of "local development" has been able to constitute a political resource, not only for the authorities but also for separatist groups. This resource has been activated in strategies of mobilisation and/or legitimation in a context marked by an identity conflict. This participative framework of development has thus been the subject of competitive appropriations, each party engaging itself in a competition for the imposition of a legitimate sense to be given to the conducted action.
\end{abstract}

